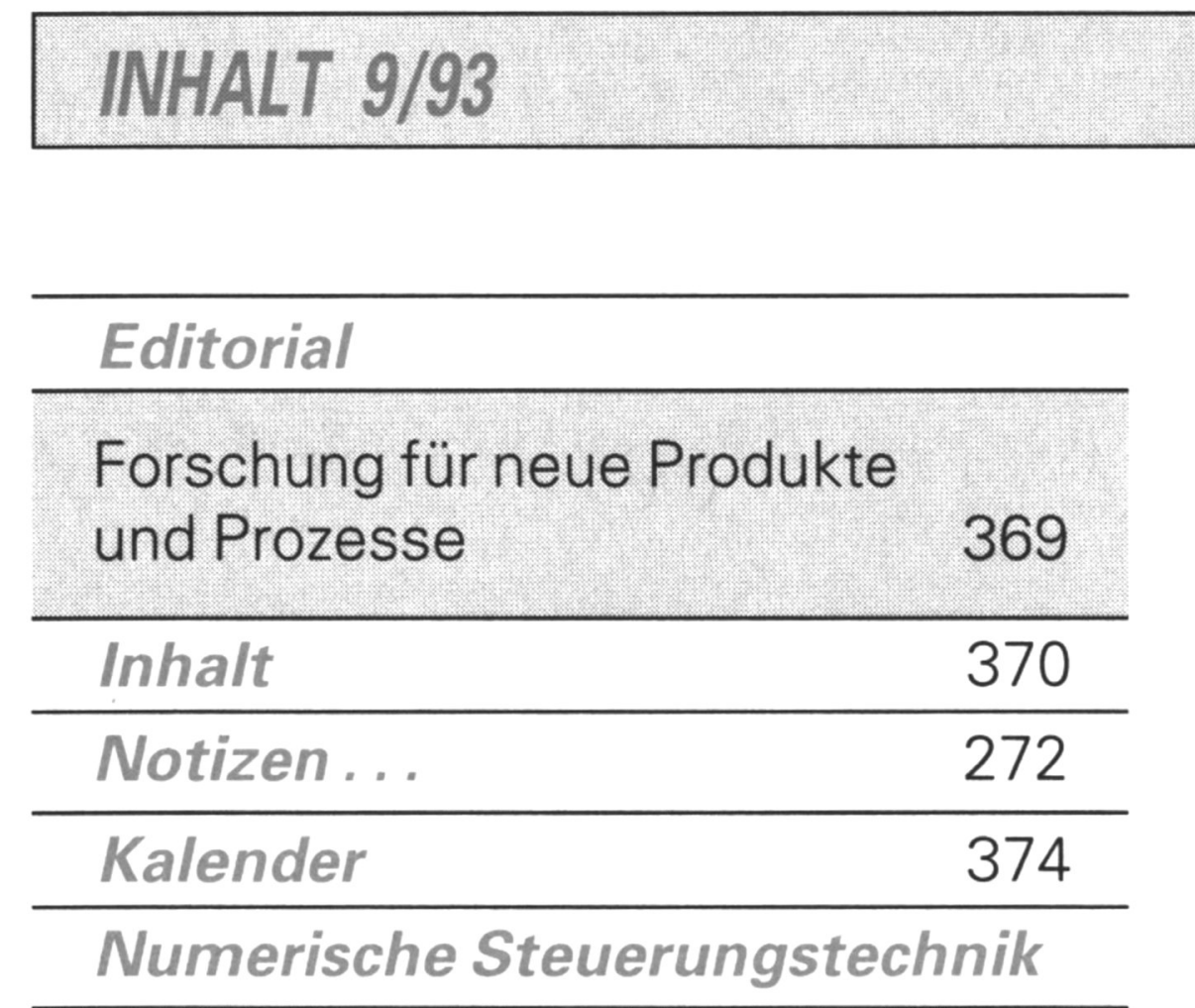

G. Spur, K. Wehmeyer

Rechnerunterstützte Fehlerkompensation an CNC-Schleifmaschinen

H. Meier

Offene Kleinsteuerung

für wettbewerbsfähige

Maschinen

CNC-Bedienoberflächen individuell gestalten

G. Spur, U. Metzler, V. Weiß WOP-Werkstückmodell als universelle

CAD/NC-Schnittstelle

PC-kompatible Maschinensteuerung

Auftragsspezifische

Schachtelplanung

Rationell fertigen mit

Gegenspindel

Zerspankraftmessung am rotierenden Werkzeug

S. Lenz, J. Barz, F. Deetz, D. Soost

Zahnrad-Profilschleifen durch

Bedienerführung und

steuerungsinterne Berechnungsmodule

Handbediente Drehmaschinen numerisch steuern

Mit hoher Leistung und Genauigkeit fräsen

G. Spur, J. Krüger, I. Suwalski, W. Straubinger, A. Heinrich CNC-integrierte Werkzeugüberwachung

U. Metzler, S. Striepe Override-Protokollierung eine neue CNC-Funktion

Werkzeuge durchgängig austauschbar

H. Hupfer, H.-J. Reddig, L. Wojcik

Anwendungsorientierte NC-Programmierung für Verdichterlaufräder
Kleinsteuerungen lösen heute durch Schnelligkeit, Analogwertverarbeitung und NC-Funktionalität eine Vielzahl von Aufgaben. Dabei besteht ein klarer Trend zur "Öffnung" der Steuerungssysteme. Hierbei sind die wichtigsten Merkmale eine standardisierte, herstellerunabhängige Programmierung, projektierbare Bedienoberflächen und Funktionalitäten, die Integration anwendungsspezifischer Module sowie standardisierte Schnittstellen. S. 378

\section{Offenes Kleinsteuerungssystem}

ZYKLUS 3

$\mathrm{V} \mathrm{m/min} 80$

U $1 / \min 3000$

\begin{tabular}{lll}
\hline Hübe & 4 \\
\hline
\end{tabular}

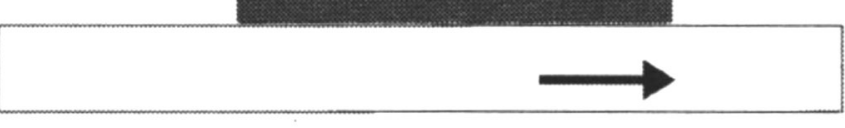

\begin{tabular}{l|l|l|l|l|} 
PREV. & MEN. & ZYKL. & ERR. & NEXT \\
\hline
\end{tabular}

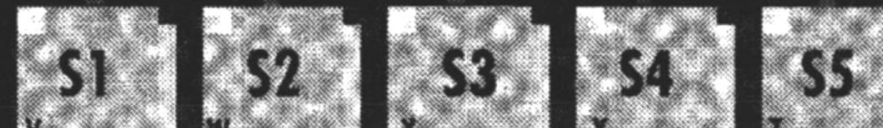

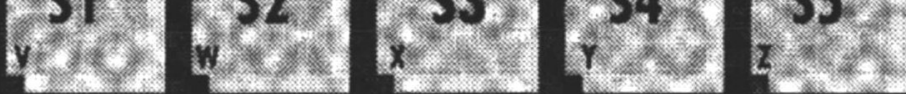
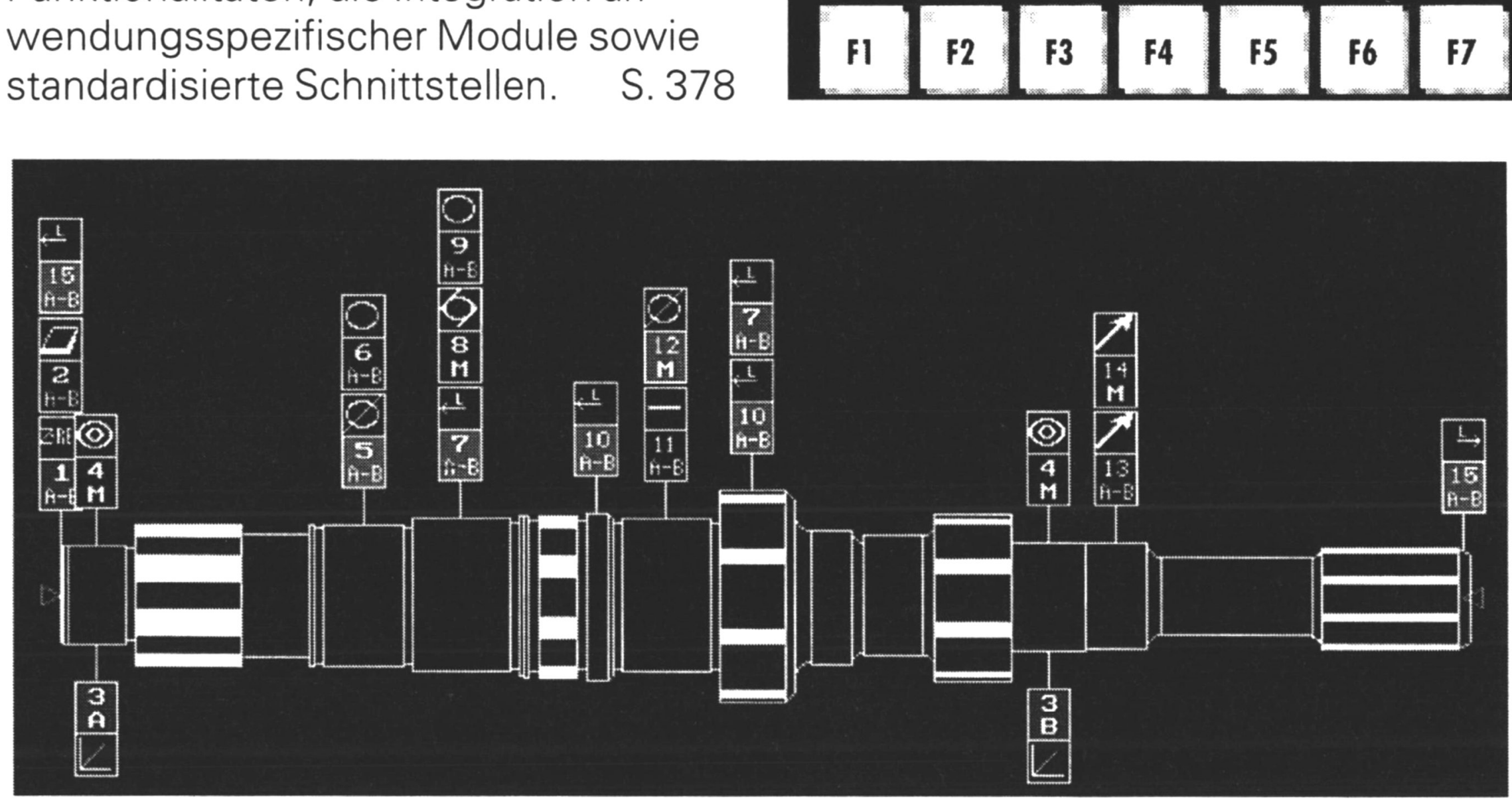

\section{Werkstattgerechte CAD-NC-Schnittstelle}

Aufgrund von Schnittstellenproblemen zwischen CAD-Systemen und NC-Programmiersystemen ist es gegenwärtig nicht möglich, alle fertigungsrelevanten Daten aus einem CAD-System für die NC-Programmierung zu nutzen. Dieser Beitrag beschreibt, wie ein WOP-Werkstückmodell mit entsprechendem Beschreibungsformat als einheitliche Schnittstelle zwischen CAD- und WOPSystemen genutzt werden kann.

S. 381

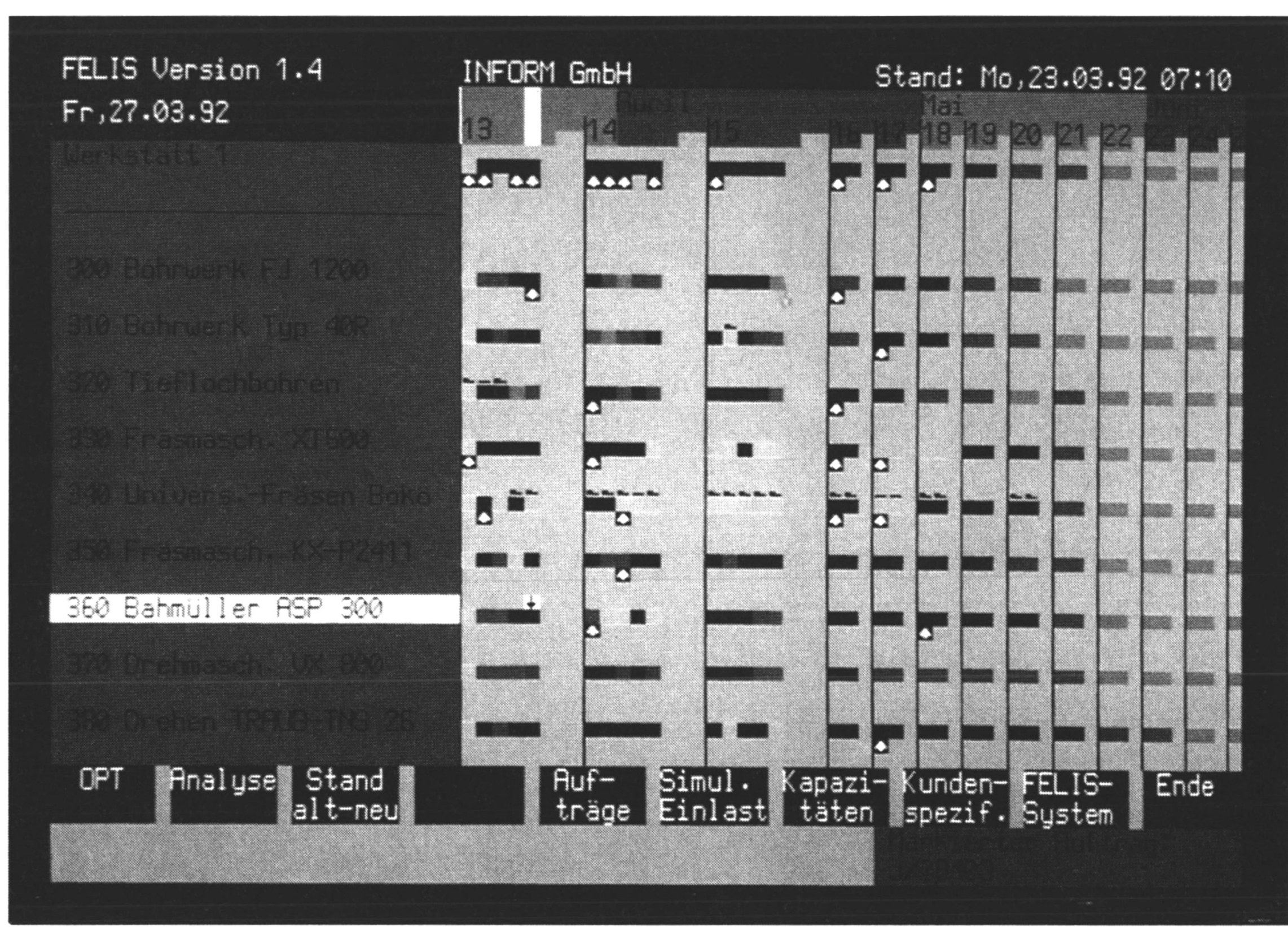

Schnell und termintreu produzieren dank unscharfer Logik

Besonders in der Einzel- und Kleinserienfertigung können Terminierungsverfahren auf der Grundlage der unscharfen Logik die Durchlaufzeiten beträchtlich verkürzen. Die hier vorgestellte Lösung mit vorausschauender Auftragsplanung, bereichsübergreifender Steuerung der Aufträge und Koordination der Fertigungsbereiche untereinander reduzierte typische Arbeitsfolge-Zeiten von durchschnittlich 2,8 auf 1,5 Tage.

S. 423 
H.-J. Pfeiffer

NC-Programme für die

Komplettbearbeitung

erstellen

Hochgeschwindigkeitsfräsen

für den Formenbau

Flexibel automatisieren mit

CNC-Systemmodulen

V. Eckhardt, J. Möller,

G. Fahrenhorst, J. Lanfermann

Graphisch-interaktive

Programmierung in der

Werkstatt

K. Rose, F. Böhle

CeA: Innovationskonzept für

Werkzeugmaschinen

mit Zukunft

M. loannides,

K.-M. Schittenhelm

Digitalisieren mit bahngeregeltem Laser-Scanner

Fertigungstechnik

G. Spur, S. J. Al-Badrawy,

J. Stirnimann

Zerspankraftmessung

bei der fünfachsigen

Fräsbearbeitung

Modularsystem für stehende und drehende Werkzeuge

Rechneranwendung

in Fertigung und Montage

A. Gladis
Zentrale Terminsteuerung mit
Fuzzy Logic

G. Pritschow, E. Wieland

Anwendungsorientiertes

Programmieren von Montagerobotern

Kundenorientiertes Steuern der Produktion

\section{In der Werkstatt programmieren}

Die bisher an Werkzeugmaschinen übliche Programmierung nach DIN 66025 läßt viele Wünsche offen. Deshalb wurde in den vergangenen Jahren mit der WOP-Initiative nach neuen Wegen der Programmierung in der Werkstatt gesucht. Dieser Beitrag beschreibt eine Programmierlösung, die eine Durchgängigkeit zwischen den Eingaben im graphisch-interaktiven System und dem DIN-66025-Programm herstellt. S. 410

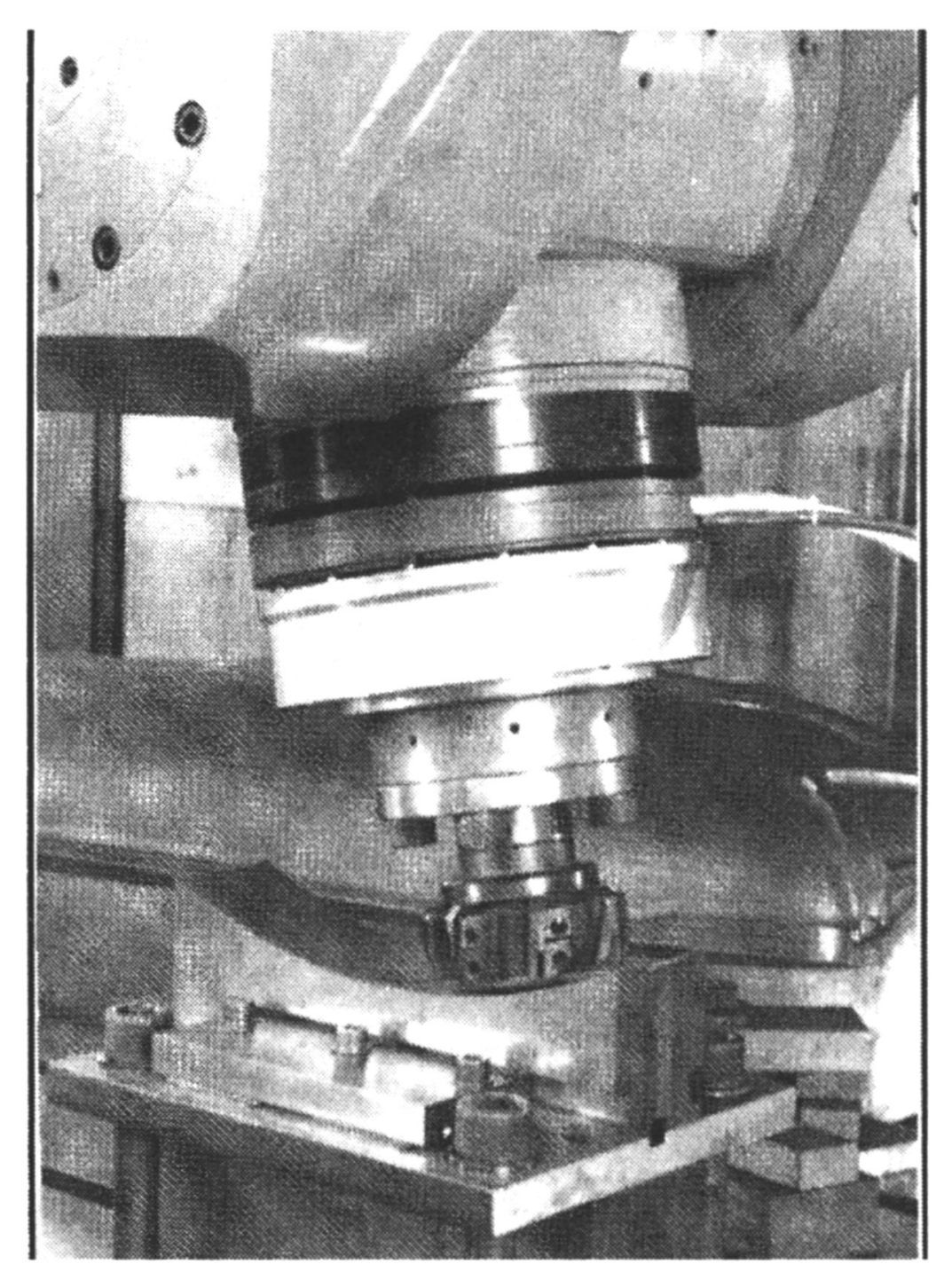

\section{Zerspankräfte beim fünfachsigen Fräsen messen}

Ein am Werkzeugschaft angebrachtes Kraftmeßsystem ermöglicht es, die an der Schneide wirkenden Kräfte $F_{t}, F_{r}$ und $F_{a}$ sowie das Moment $M_{t}$ ohne Transformation vom Werkstück-Koordinatensystem in das rotierende Werkzeug-Koordinatensystem zu erfassen. Mit Hilfe dieses Kraftmeßsystems wurden bei zerspantechnischen Untersuchungen direkte Aussagen über die auf der Schneide wirkenden Kräfte bei der fünfachsigen Fräsbearbeitung von konvexen und konkaven Flächen gemacht.

S. 419

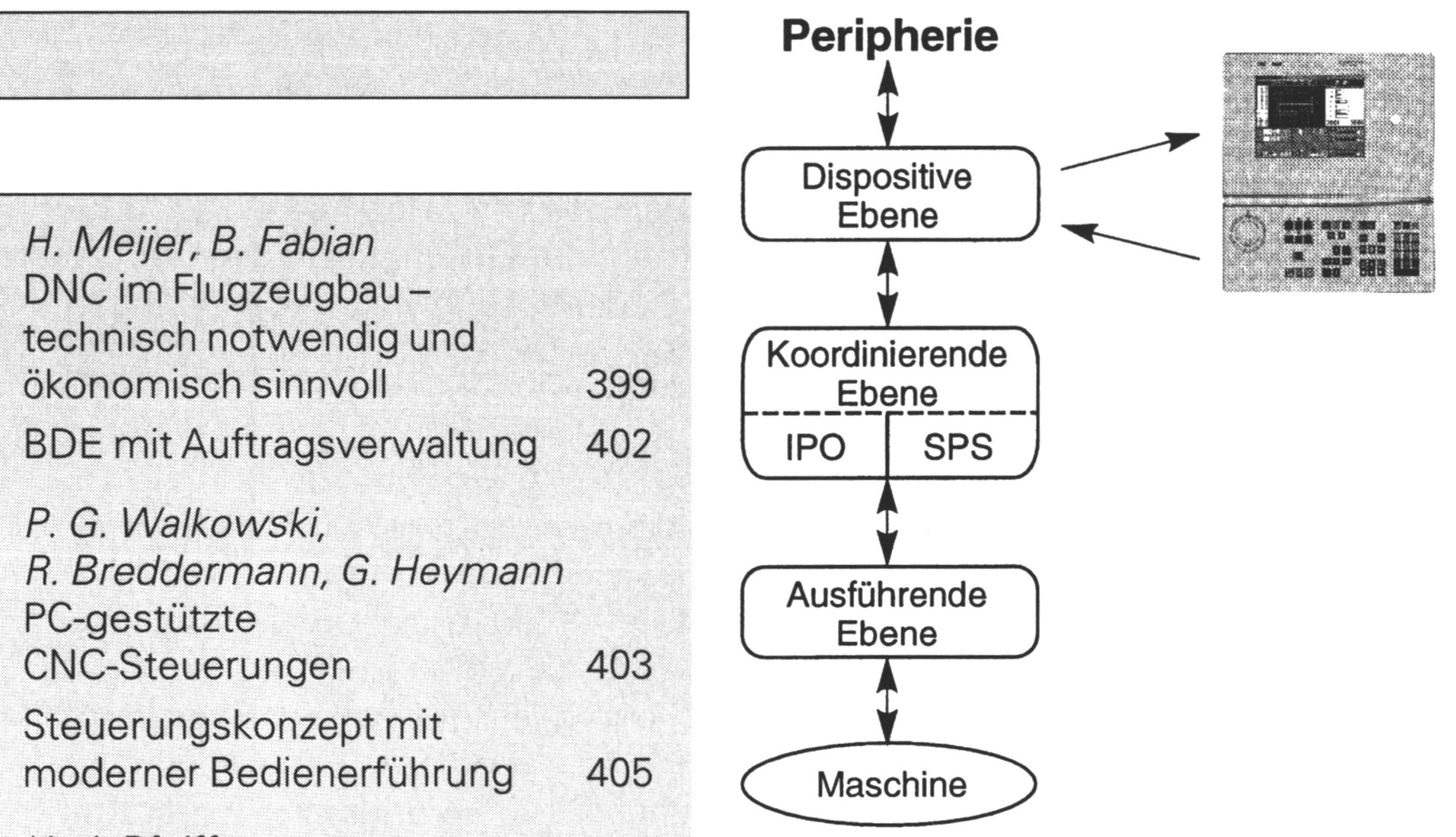

INHALT 9/93

Fabrikanlagen und Materialfluß

K. Lang

Systematisches Entwickeln von Werkzeugmachinen

H. K. Tönshoff, L. Barfels

Zentralisierte Teilefertigung

im Unternehmensverbund

Die Einsatzschwelle von Luftlagern sinkt

FTS automatisch und manuell einsetzbar

Objekterkennung mit induktiven Identifikationssystemen

Neue Plattformtechnik

fördert Teamarbeit

\begin{tabular}{lr}
\hline Report & \\
\hline $\begin{array}{l}\text { Fabrikanlagen und } \\
\text { Materialfluß }\end{array}$ & 438 \\
CAD C CAM C CIM & 440 \\
\hline Impressum & 440 \\
\hline Supplement: & \\
Laser-Praxis & LS 55-LS 102 \\
\hline
\end{tabular}

R. Florian

Der Laser hat seine

Grenzen

\section{K. Stratmann}

Kombinieren rechnet sich

U. Schulz

Der Strahl liegt immer richtig

LS 86

Supplement:

Der Zuliefermarkt ZM 129-ZM 188

T. Lauenstein

Schraubenfertigung ganz-

heitlich modernisieren

ZM 137

D. Meyer

Spritzgießfertigung

automatisieren

ZM 144

J. Haunschmid,

D. Wachholder

Mülltonnen mit Recyclat gebrauchstüchtig und langlebig

W. Behrens

Foggingverhalten von KFZInnenausstattungen

K.-H. Wierz

Gehäusebau - Blechverarbeitung und mehr

W. Lutz

Auf den Sockel gehoben ZM 160 